Canadian

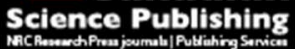

Canadian Journal of Microbiology Revue canadienne de de microbiologie

IS256 Abolishes gelatinase activity and biofilm formation in a mutant of the nosocomial pathogen Enterococcus faecalis V583

\begin{tabular}{|r|l|}
\hline Journal: & Canadian Journal of Microbiology \\
\hline Manuscript ID: & cjm-2015-0090.R1 \\
\hline Danuscript Type: & Note \\
\hline Complete List of Authors: & $\begin{array}{l}\text { Perez, Marta; IPLA-CSIC, Technology and biotechnology of dairy products } \\
\text { Calles-Enríquez, Marina; IPLA-CSIC, Technology and biotechnology of dairy } \\
\text { products } \\
\text { del Rio, Beatriz; IPLA-CSIC, Technology and biotechnology of dairy } \\
\text { products } \\
\text { Ladero, Victor; IPLA-CSIC, Technology and biotechnology of dairy products } \\
\text { Martin, M Cruz; IPLA-CSIC, Technology and biotechnology of dairy } \\
\text { products } \\
\text { Fernandez, Maria; IPLA-CSIC, Technology and biotechnology of dairy } \\
\text { products } \\
\text { Alvarez, Miguel; IPLA-CSIC, Technology and biotechnology of dairy } \\
\text { products }\end{array}$ \\
\hline Keyword: & \begin{tabular}{l} 
Enterococcus faecalis, insertion sequence, biofilm, gelatinase \\
\hline
\end{tabular} \\
\hline
\end{tabular}




\section{IS256 Abolishes Gelatinase Activity and Biofilm Formation in a Mutant of the} Nosocomial Pathogen Enterococcus faecalis V583

Marta Perez, Marina Calles-Enríquez, Beatriz del Río, Victor Ladero, María Cruz Martín, María Fernández* and Miguel A. Alvarez

Instituto de Productos Lácteos de Asturias (IPLA-CSIC), Paseo Río Linares s/n, 33300 Villaviciosa, Asturias, Spain.

E-mail addresses:

Marta Perez: mpgarcia@ipla.csic.es

Marina Calles-Enríquez: marynacalles@hotmail.com

Victor Ladero: ladero@ipla.csic.es

Beatriz del Rio: beadelrio@ipla.csic.es

Ma Cruz Martin: mcm@ipla.csic.es

Miguel A. Alvarez: maag@ipla.csic.es

* Corresponding author: María Fernández.

e-mail: mfernandez@ipla.csic.es

Tel.: +34 9858921 31; fax: +34 985892233 . 


\begin{abstract}
Enterococcus faecalis is one of the most controversial species of lactic acid bacteria. Some strains are used as probiotic, while others are associated with severe and life threatening nosocomial infections. Their pathogenicity depends on the acquisition of multi drug resistance and virulence factors. Gelatinase, which is required in the first steps of biofilm formation, is an important virulence determinant involved in E. faecalis pathogenesis including endocarditis and peritonitis. The gene that codes for gelatinase ( $\mathrm{gelE}$ ) is controlled by the Fsr quorum-sensing system, whose encoding genes ( $f s r A$, $f_{s r} B, f_{s r} C$, and $\left.f_{s r} D\right)$ are located immediately upstream of gelE.

The integration of a DNA fragment into the $f s r$ locus of a derived mutant of E. faecalis V583 suppressed the gelatinase activity and prevented biofilm formation. Sequence analysis indicated the presence of IS256 integrated into the $f_{s r} C$ gene at nucleotide position 321. Interestingly, IS256 is also associated with biofilm formation in Staphylococcus epidermidis and Staphylococcus aureus. This is the first description of an insertion sequence that prevents biofilm formation in E. faecalis.
\end{abstract}

Key words: Enterococcus faecalis, insertion sequence, biofilm, gelatinase 
Enterococcus faecalis, a Gram-positive bacterium, is generally thought to be a commensal of the mammalian gastrointestinal tract. However, its ability to endure a range of harsh conditions allows it survive in other environments. It is also an opportunistic pathogen that can cause nosocomial infections, including bacteremia and biofilm-based pathogeneses such as endocarditis and urinary tract infections (Paulsen et al. 2003). The problem of hospital-acquired enterococcal infections has been aggravated in recent decades due to the alarming increase in emergent vancomycin-resistant enterococci (VRE) (Pan et al. 2012).

Gelatinase, an extracellular protease, is known to be involved in E. faecalis pathogenesis, and is synthesized by approximately $60 \%$ of clinical isolates (GallowayPena et al. 2011). It is required in the first steps of biofilm formation (Hancock and Perego 2004) and contributes towards virulence by hydrolyzing host substrates such as collagen, fibrinogen, fibrin, endothelin-1, bradykinin, LL-37 and complement components C3 and C3a (Thurlow et al. 2010).

The genes that code for gelatinase ( $g e l E)$ and serine protease ( $\operatorname{sprE}$ ) form an operon controlled by the Fsr quorum-sensing system located immediately upstream of gelE. The two-component system of enterococcal Fsr includes $f_{s r} A$ (a response regulator), $f_{s r} B$ (a propeptide processing protein), $f_{s r} C$ (histidine kinase), and $f_{s r} D$ (a gelatinase biosynthesis activating pheromone [GBAP]) (Nakayama et al. 2006). The Fsr system of E. faecalis is required for the production of gelatinase (Hancock and Perego 2004), and appears to be involved in the regulation of other genes important in virulence and metabolism (Bourgogne et al. 2006; Teixeira et al. 2013).

This paper describes the effect of the integration of IS into the $f_{s r} C$ gene in a laboratory derivative mutant of E. faecalis strain V583. 
E. faecalis V583 was isolated in 1987 from a blood culture of a chronicallyinfected patient. It was the first clinical VRE isolate reported from the United States (Sahm et al. 1989) and Shankar et al., (2002) demonstrated that variations within the structure of the pathogenicity islands modulate the virulence of this stain. During the construction of an E. faecalis V583 non-tyramine-producing mutant (Perez et al. 2014) by double-crossover deletion (Jonsson et al. 2009) of the tyrosine decarboxylase $(t d c)$ gene cluster (Ladero et al. 2012), it was observed that one of the three independent $E$. faecalis V583 $\Delta t d c$ mutants obtained did not produce gelatinase. This was confirmed by streaking single colonies on M17 agar plates containing $30 \mathrm{gL}^{-1}$ of gelatin and incubating overnight at $37^{\circ} \mathrm{C}$ (Reviriego et al. 2005).

The primers fsrA1F (5'-GCAGGAAACTACTGAAATCGC-3') and sprE1R (5'CTCGAGATTTCCCGTGATTCTGG-3') were designed based on E. faecalis V583 genome sequence (GenBank accession number: AE016830) to PCR- amplify the $f s r A B D C$-gelE-sprE locus. The wild type strain and the $\Delta t d c$ mutants yielded the expected $5767 \mathrm{bp}$ fragment. However, the amplification of the strain unable to produce gelatinase resulted in a $7091 \mathrm{bp}$ amplicon (data not shown). New primers were designed in order to sequence this unexpected fragment: fsrB1F (5'GTGCAATACTTGAAGAGGAGGG-3'), fsrC1R (5'-CATATAACAATCC CCAACCGTGC-3'), fsrC1F (5'-GATAACAAATAGTGTCCAAGCCG-3'), gelE1R (5'-CATAAGATTATGCCACTCCTTATCC-3'), fsrC2R (5'-TCATCATGTAGGTCC ATAAGAACGGC-3') and fsrC2R (5'-CGTAAAGCTGCGCTCATAATAGCC-3'). Sequence analysis (performed by Macrogen, Korea) indicated the presence of a $1324 \mathrm{bp}$ DNA fragment integrated into the $f_{s r} C$ gene at nucleotide position 321 . The insert corresponded to IS256. This was orientated in the same transcriptional direction as the 
$f_{s r}$ genes and had a target site duplication of $8 \mathrm{bp}$ in the flanked regions (Figure 1). The nucleotide sequence of the $f$ sr $A B C D$ locus containing IS256 was deposited in the European Nucleotide Archive under accession number HG794359.

The construction of a $f s r C$ mutant strain and their subsequent complementation demonstrated that $f_{s r} C$ expression is necessary for gelE expression and biofilm production (Hancock and Perego 2004), experiments were performed to determine whether the IS256 in the frs $C$ gene affected the capacity of the strain to form a biofilm on polystyrene microtiter plates (TC Microwell 96U, Thermo Scientific, Denmark). Briefly, E. faecalis strains were grown overnight in M17 medium with $14 \mathrm{mM}$ glucose at $37^{\circ} \mathrm{C}$. The culture was diluted 1;40 in $200 \mu \mathrm{l}$ of the same media. The microtiter plates were incubated at $37^{\circ} \mathrm{C}$ for $24 \mathrm{~h}$ in aerobic conditions. The cells were washed, stained with crystal violet and the optical density was determined following a method previously described (Hancock and Perego 2004). The capacity of the gelatinasenegative strain to form biofilms was much reduced compared to the parental strain and $\Delta t d c$ gelatinase-positive mutants (Figure 2).

These results suggest that the insertion of IS256 into $f s r C$ leads to a truncated histidine kinase. This would lead to impaired GBAP signalling, therefore preventing the expression of gelE and biofilm formation.

It has been indicated the high plasticity of the E. faecalis genome in the area of the Fsr system (Galloway-Pena et al. 2011). The gelatinase-negative phenotype has been reported for both natural and laboratory E. faecalis strains (Teixeira et al. 2012). It has a number of genetic causes, mostly involving the $f s r$ locus (Shankar et al. 2012). However, this is the first report of an IS causing gelE not to be expressed. 
IS256, which encodes a transposase, is widespread in the genomes of multiresistant staphylococci and enterococci. The sequence appears as multiple free copies as well as forming the ends of the composite aminoglycoside resistance-mediating transposon Tn4001 (Hennig and Ziebuhr 2010). E. faecalis V583 has multiple copies of IS256 in its genome, 6 in the chromosome and 4 spread across 3 plasmids (Paulsen et al. 2003).

IS256 has been identified as a marker of multidrug resistance and biofilmformation in clinical isolates of staphylococci (Hennig and Ziebuhr 2010). It is associated with biofilm formation via its reversible transposition into the ica operon in S. epidermidis (Ziebuhr et al. 1999) and S. aureus (Valle et al. 2007), and into the agr operon in S. aureus (Cafiso et al. 2007). Biofilm-associated genes and regulators seem to be an important hot-spot for IS256 integration in staphylococci. As shown by the present results, IS256 is also associated with biofilm-forming genes in enterococci.

It has been shown that a temperature of $30^{\circ} \mathrm{C}$ favors the transposition of IS 256 in S. aureus (Valle et al. 2007). The present production of E. faecalis mutants by homologous recombination (Jonsson et al. 2009) involved growth at $28^{\circ} \mathrm{C}$ for approximately 75 generations, which may have similarly favored the transposition process.

It is difficult to speculate upon the survival/evolutionary benefit that silencing the Fsr system would bring. In any event, minority subpopulations of E. faecalis GBAP quorum non-responders - perhaps arising from accumulation of mutations in the $f_{s r}$ locus - have been reported (Thomas et al. 2009). Thus, the transposition of IS256 might provide a mechanism for the generation of GBAP non-responders, which might have an evolutionary advantage under certain conditions. It has been suggested that, in 
staphylococci, the shutdown of biofilm formation by IS256 may help dissemination into novel habitats (Ziebuhr et al. 1999).

Given the clinical importance of biofilm formation, and of gelatinase as a promising target for therapeutic intervention against multidrug-resistant and virulent $E$. faecalis strains, further investigations should be performed to unravel the complex regulation of the Fsr system. The genetic causes of phenotypic change in biofilmforming ability should be determined, taking into account the importance of mobile elements in genome flexibility, adaptation and evolution.

\section{Acknowledgements}

This work was funded by the Spanish Ministry of Economy and Competitiveness (AGL2013-45431-R). M.P. was the recipient of an FPU fellowship from the Spanish Ministry of Education, Culture and Sport. The authors thank Adrian Burton for linguistic assistance.

\section{References}

Bourgogne, A., Hilsenbeck, S.G., Dunny, G.M., and Murray, B.E. 2006. Comparison of OG1RF and an isogenic $f_{s r} B$ deletion mutant by transcriptional analysis: the Fsr system of Enterococcus faecalis is more than the activator of gelatinase and serine protease. $\mathrm{J}$ Bacteriol 188(8): 2875-2884.

Cafiso, V., Bertuccio, T., Santagati, M., Demelio, V., Spina, D., Nicoletti, G., and Stefani, S. 2007. agr-Genotyping and transcriptional analysis of biofilm-producing Staphylococcus aureus. FEMS Immunol Med Microbiol 51(1): 220-227. 
Galloway-Pena, J.R., Bourgogne, A., Qin, X., and Murray, B.E. 2011. Diversity of the

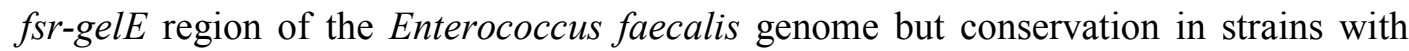
partial deletions of the fsr operon. Appl Environ Microbiol 77(2): 442-451.

Hancock, L.E., and Perego, M. 2004. The Enterococcus faecalis fsr two-component system controls biofilm development through production of gelatinase. J Bacteriol 186(17): 5629-5639.

Hennig, S., and Ziebuhr, W. 2010. Characterization of the transposase encoded by IS256, the prototype of a major family of bacterial insertion sequence elements. J Bacteriol 192(16): 4153-4163.

Jonsson, M., Saleihan, Z., Nes, I.F., and Holo, H. 2009. Construction and characterization of three lactate dehydrogenase-negative Enterococcus faecalis V583 mutants. Appl Environ Microbiol 75(14): 4901-4903.

Ladero, V., Fernandez, M., Calles-Enriquez, M., Sanchez-Llana, E., Canedo, E., Martin, M.C., and Alvarez, M.A. 2012. Is the production of the biogenic amines tyramine and putrescine a species-level trait in enterococci? Food Microbiol 30(1): 132-138.

Nakayama, J., Chen, S., Oyama, N., Nishiguchi, K., Azab, E.A., Tanaka, E., et al. 2006. Revised model for Enterococcus faecalis fsr quorum-sensing system: the small open reading frame $f_{s r} D$ encodes the gelatinase biosynthesis-activating pheromone propeptide corresponding to staphylococcal AgrD. J Bacteriol 188(23): 8321-8326.

Pan, S.C., Wang, J.T., Chen, Y.C., Chang, Y.Y., Chen, M.L., and Chang, S.C. 2012. Incidence of and risk factors for infection or colonization of vancomycin-resistant enterococci in patients in the intensive care unit. PLoS One 7(10): e47297. 
Paulsen, I.T., Banerjei, L., Myers, G.S., Nelson, K.E., Seshadri, R., Read, T.D., et al. 2003. Role of mobile DNA in the evolution of vancomycin-resistant Enterococcus faecalis. Science 299(5615): 2071-2074.

Perez, M., Calles-Enríquez, M., Ingolf, N., Martin, M.C., Fernandez, M., Ladero, V., and Alvarez, M.A. 2014. Tyramine biosynthesis is transcriptionally induced at low $\mathrm{pH}$ and improves the fitness of Enterococcus faecalis in acidic environments. Applied Microbiology and Biotechnology.

Reviriego, C., Eaton, T., Martin, R., Jimenez, E., Fernandez, L., Gasson, M.J., and Rodriguez, J.M. 2005. Screening of virulence determinants in Enterococcus faecium strains isolated from breast milk. J Hum Lact 21(2): 131-137.

Sahm, D.F., Kissinger, J., Gilmore, M.S., Murray, P.R., Mulder, R., Solliday, J., and Clarke, B. 1989. In vitro susceptibility studies of vancomycin-resistant Enterococcus faecalis. Antimicrob Agents Chemother 33(9): 1588-1591.

Shankar, N., Baghdayan, A.S., and Gilmore, M.S. 2002. Modulation of virulence within a pathogenicity island in vancomycin-resistant Enterococcus faecalis. Nature. 13(6890):746-750

Shankar, J., Walker, R.G., Ward, D., and Horsburgh, M.J. 2012. The Enterococcus faecalis exoproteome: identification and temporal regulation by Fsr. PLoS One 7(3): e33450.

Teixeira, N., Santos, S., Marujo, P., Yokohata, R., Iyer, V.S., Nakayama, J., et al. 2012. The incongruent gelatinase genotype and phenotype in Enterococcus faecalis are due to 
shutting off the ability to respond to the gelatinase biosynthesis-activating pheromone (GBAP) quorum-sensing signal. Microbiology 158(Pt 2): 519-528.

Teixeira, N., Varahan, S., Gorman, M.J., Palmer, K.L., Zaidman-Remy, A., Yokohata, R., et al. 2013. Drosophila host model reveals new Enterococcus faecalis quorumsensing associated virulence factors. PLoS One 8(5): e64740.

Thomas, V.C., Hiromasa, Y., Harms, N., Thurlow, L., Tomich, J., and Hancock, L.E. 2009. A fratricidal mechanism is responsible for eDNA release and contributes to biofilm development of Enterococcus faecalis. Mol Microbiol 72(4): 1022-1036.

Thurlow, L.R., Thomas, V.C., Narayanan, S., Olson, S., Fleming, S.D., and Hancock, L.E. 2010. Gelatinase contributes to the pathogenesis of endocarditis caused by Enterococcus faecalis. Infect Immun 78(11): 4936-4943.

Valle, J., Vergara-Irigaray, M., Merino, N., Penades, J.R., and Lasa, I. 2007. sigmaB regulates IS256-mediated Staphylococcus aureus biofilm phenotypic variation. J Bacteriol 189(7): 2886-2896.

Ziebuhr, W., Krimmer, V., Rachid, S., Lossner, I., Gotz, F., and Hacker, J. 1999. A novel mechanism of phase variation of virulence in Staphylococcus epidermidis: evidence for control of the polysaccharide intercellular adhesin synthesis by alternating insertion and excision of the insertion sequence element IS256. Mol Microbiol 32(2): $345-356$. 


\section{Legend of figures}

Figure 1.

Diagram showing the IS256 insertion. IS256 was inserted into the $f s r$ locus of the $E$. faecalis V583 $\Delta t d c$ gelatinase-negative strain.

Figure 2.

Biofilm formation on polystyrene microtiter plates. 1. E. faecalis V583 parental strain; 2. E. faecalis V583 $\Delta t d c$ strain; 3. E. faecalis V583 $\Delta t d c$ gelatinase-negative strain. The asterisk indicates statistically significant difference $(P \leq 0.001$; Student's $t$-test $)$ in comparison to the other conditions. 


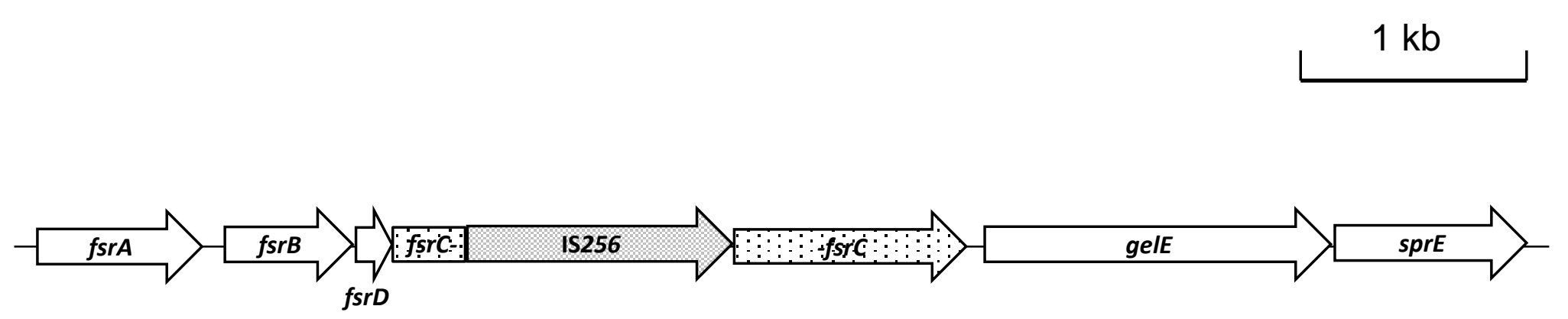

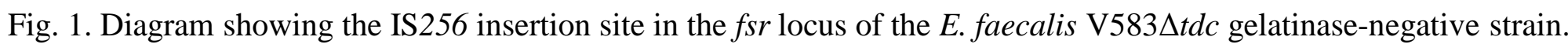




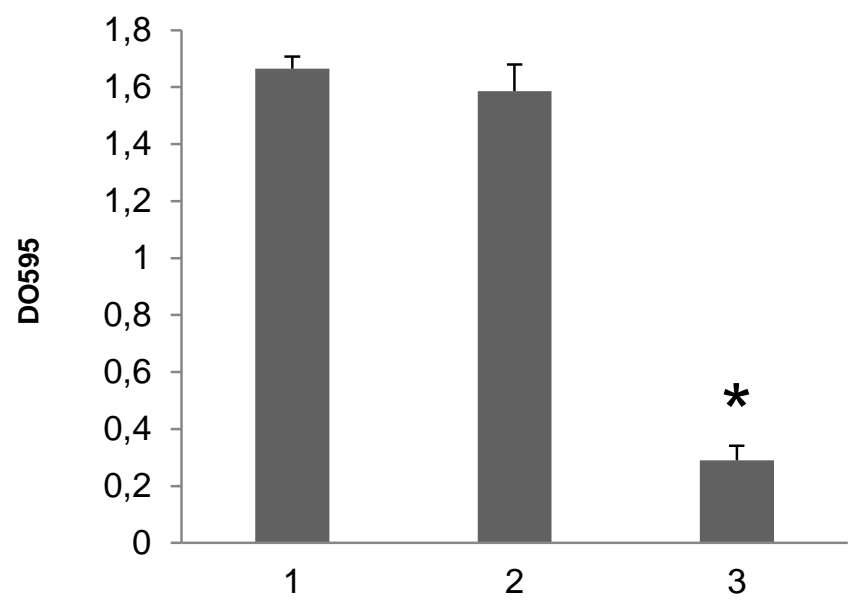

Figure 2

https://mc06.manuscriptcentral.com/cjm-pubs 